\title{
Electrochemical Synthesis of Silver Nanoparticles and their Potential Use as Antimicrobial Agent: a Case Study on Escherichia Coli
}

\author{
Lucas Blandón, ${ }^{a, *}$ Mario V. Vázquez, ${ }^{a}$ Dora M. Benjumea ${ }^{b}$ and Gelmy Ciro ${ }^{b}$ \\ ${ }^{a}$ Grupo interdisciplinario de estudios moleculares GIEM. Universidad de Antioquia \\ calle 70 No. 52 - 27.Medellín, Antioquia, Colombia \\ ${ }^{b}$ Programa de Ofidismo y Escorpionismo. Universidad de Antioquia Calle 62 \# 52-59 \\ Medellín, Antioquia, Colombia
}

Received 27 February 2012; accepted 30 April 2012

\begin{abstract} are satisfactory.

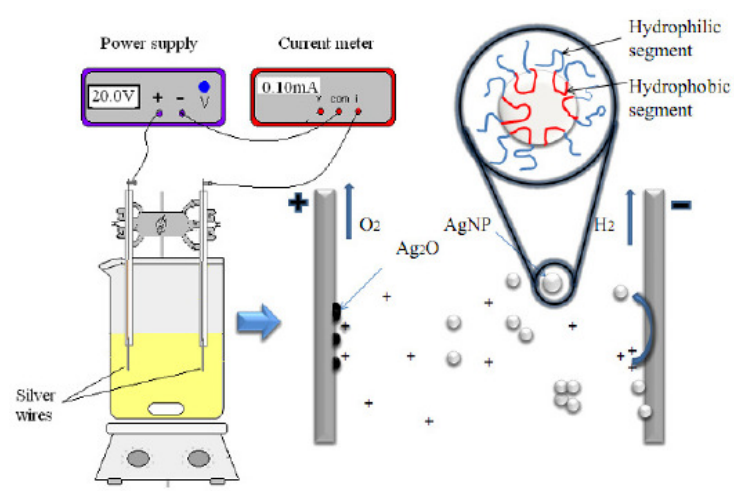

Silver nanoparticles (AgNP) were synthesized by electrochemical method using high purity metallic silver in the presence of the surfactant Pluronic ${ }^{\circledR}$ f127. The synthesized nanoparticles were characterized using UV-Vis spectroscopy, Dynamic Light Scattering (DLS), X-Ray Dispersion Spectroscopy (XDS) and Scanning Electron Microscopy (SEM). The antimicrobial activity of AgNP was evaluated against Escherichia Coli.

In presence of surfactant, the prepared nanoparticles led to a monomodal distribution with an average size less than $100 \mathrm{~nm}$. The minimal inhibitory concentration (MIC) of the resulting suspension was evaluated using micro dilution method and the MIC results

Keywords: electrosynthesis, silver nanoparticles, surfactant, antimicrobial agent.

\footnotetext{
* Corresponding author. E-mail: blandonn@une.net.co
} 


\section{Introduction}

Since last decades, the field of metallic nanomaterials has been of great interest and scientific development due to their physical and chemical properties. Therefore, these are widely used in electronic, optic, catalysis and antimicrobial areas [1-3]. The last application has attracted considerable interest in relation with methods and uses of AgNP, which have proven quite effective growth inhibition of microorganism. The antimicrobial activity of AgNP has been evaluated in different bacteria such as Escherichia coli [4], Streptococcus mutans [5], Streptococcus pyogenes, Salmonella typhi, Klebsiella pneumoniae, Vibrio cholerae [6], Salmonella aureus and Bacillus subtilis [7]. Although the antibacterial activity mechanism is unclear, some studies using techniques, such as SEM and atomic force microscopy, suggest that AgNP acts on the cellular membrane surface, affecting the permeability, the respiratory functions and preventing the cell division which leads to cellular death $[7,8]$.

To assess the antimicrobial activity of AgNP, different methods can be used to determine the growth inhibiting of in vitro cultures of microorganism. These tests may be qualitative or quantitative. In the last case, the main interest is to calculate the MIC, which is the lowest concentration where the antimicrobial agent inhibits the growth of the microorganism $[4,8,9]$. One of the most used methods for calculation of MIC has been published by Abate and coworkers [10], where the MIC is determined by a colorimetric semi quantitative technique.

Concerning the AgNP synthesis methods, several of them are based on physical processes such as laser ablation, milling and thermal decomposition, and chemical methods, which consist basically in the chemical reduction of $\mathrm{Ag}^{+}$ions. These syntheses are carried out in organic or aqueous solvents and the use of reducing agents such as sodium borohydride, citrate, ascorbate and some carbohydrates (fructose and glucose) has been reported [8,11,12].

Another proposal for the AgNP synthesis are the electrochemical methods. These methods have been implemented for the synthesis of metallic nanoparticles due to the advantages in experimental conditions control. In 1994, Reetz and Heilbig [13] published a work with a detailed description for the electrochemical synthesis, in which a metal plate was anodically dissolved and the resulting salt was reduced in a cathode, yielding particles stabilized with tetraalkylammonium salts which in turn meet electrolyte function. Other authors, had proposed electrochemical methods for the synthesis of AgNP using other types of electrolytes like ethanol: $\mathrm{KNO}_{3}[14]$, poly(N-vinylpyrrolidone) [15] and deionized water [16].

For most applications of metallic nanoparticles it is required to make superficial modifications in order to promote the suspension stability. In this regard, ionic and neutral surfactants have been used including PEG derivates, oligo and polysaccharides, and triblock co-polymers type poly(ethylene oxide)poly(propylene oxide)-poly(ethylene oxide) known as Pluronics®, which are biocompatible and their hydrophobic-hydrophilic character is given by the relation between ethylene oxide and propylene oxide [17]. Another important ratio is the relationship between nanoparticles and surfactant concentrations. If this ratio is high, it can lead to non-stabilized particles that produce flocculation 
and growth of the nanoparticle diameter. On the other hand, if the concentration of surfactant is much more higher than the one of the nanoparticles, phenomena, such as depletion flocculation can occur [18]. However this phenomenon is not a chemical interaction and its formation energies aren't high, so it is a reversible system.

This paper assesses the antimicrobial activity of AgNP synthesized by an electrochemical method from metallic silver and in the presence of Pluronic ${ }^{\circledR}$ f127 as a surfactant. The synthesized nanoparticles are characterized using UVVisible spectroscopy, X-ray dispersion spectroscopy (XDS), dynamic light scattering (DLS), and scanning electron microscopy (SEM).

\section{Materials and methods}

\section{Electrochemical synthesis}

Two identical silver wires with a purity of $99.95 \%$ and geometric area of $74 \mathrm{~mm}^{2}$ were used for the electrochemical synthesis. The wires were submerged in deionized water or in the presence of Pluronic ${ }^{\circledR}$ f127 at concentrations of $4.5 \mathrm{x}$ $10^{-4}, 1 \times 10^{-4}$ and $1 \times 10^{-5} \mathrm{M}$ all these below the critical micellar concentration. A differential potential of $20 \mathrm{~V}$ was applied between the electrodes, changing the polarity every four minutes, holding the system under constant magnetic stirring, and at room temperature, as reported by Khaydarov and coworkers [16]. The potential was applied with a controlled potential power supply BK PRECISION® 1666 and the generated current was measured over time using a Fluke 45 Dual Display meter connected in series with the system. After reaction, a drop of $\mathrm{H}_{2} \mathrm{O}_{2}$ $30 \%(\mathrm{v} / \mathrm{v})$ was added to reduce all silver oxide formed as byproduct. The synthesized particles were passed through a $0.2 \mu \mathrm{m}$ filter and then proceeded to the characterization.

\section{AgNP characterization}

UV-Vis spectroscopy was used to monitor the reaction and to characterize the AgNP. DLS was used to determine the particle size distribution (PSD) and XDS to define the composition, while SEM allowed the evaluation of the morphology of the AgNP.

The spectroscopic study was carried out in a Jenway $640 \mathrm{UV} / \mathrm{V}$ is spectrophotometer sampling every twelve minutes. The PSD was measured in a Horiba DLS particle size analyzer. The SEM and EDX analyses were run in a JEOL JSM-6490LV microscopy coupled with a microanalysis EDX system.

\section{Antimicrobial evaluation}

A colorimetric broth micro dilution method proposed by Abate et al. [10], with some modification, was used in the evaluation of the Minimum Inhibitory Concentration (MIC) of the AgNP. Diluted solutions of the AgNP were prepared. The concentration of AgNP ranged from 0.03 to $16 \mathrm{mg} / \mathrm{L}$ and was placed in 96well microplates (Becton Dickinson Labware ${ }^{\circledR}$, USA). Then, Muller-Hinton broth (Merck®, Germany) and the microorganism (Escherichia coli ATCC 25922 ), in the exponential growth phase (approximately $1.5 \times 10^{8} \mathrm{CFU} / \mathrm{mL}$ ), 
were added to each of the wells. After incubation $\left(37 \pm 0.2{ }^{\circ} \mathrm{C} / 5 \mathrm{~h}\right)$, a tetrazolium salt solution $(0.8 \mathrm{mg} / \mathrm{mL})$, also known as MTT (3-\{4.5-dimethylthiazol-2-yl $\}$ 2,5-diphenyl tetrazolium bromide) (Alfa Aesar $\AA$, Germany), was added to each of the wells and subjected to an incubation process at $37 \pm 0.2{ }^{\circ} \mathrm{C}$ for $1 \mathrm{~h}$ in order to allow the viable microorganisms to metabolize the yellow dye MTT into formazan (purple crystals) (26-30). The MIC value was considered as the concentration of the first well that did not undergo color change (from yellow to purple). The procedure was repeated three times for each microorganism. After determining the MIC, the wells, whose microorganism did not experience any growth, were transferred to a solid medium. After $24 \mathrm{~h}$ of incubation, the concentration at which there was not microorganism growth was considered as the minimum bactericidal concentration against $E$. coli.

The concentration of AgNP in suspension was calculated theoretically assuming a $100 \%$ current efficiency for nanoparticles synthesis.

\section{Results and discussion}

\section{Electrochemical synthesis}

Fig. 1 shows the current-time profile obtained for AgNP synthesis. This figure shows that the initial current is low and increases over time, which corresponds with the highly resistive medium used for the reaction. As the reaction progresses, the current increases progressively because of the anodic oxidation of wire $\mathrm{Ag}^{+}$ions from the wire. After 50 minutes, the current begins to stabilize indicating that the system reaches equilibrium for the oxidized and reduced silver.

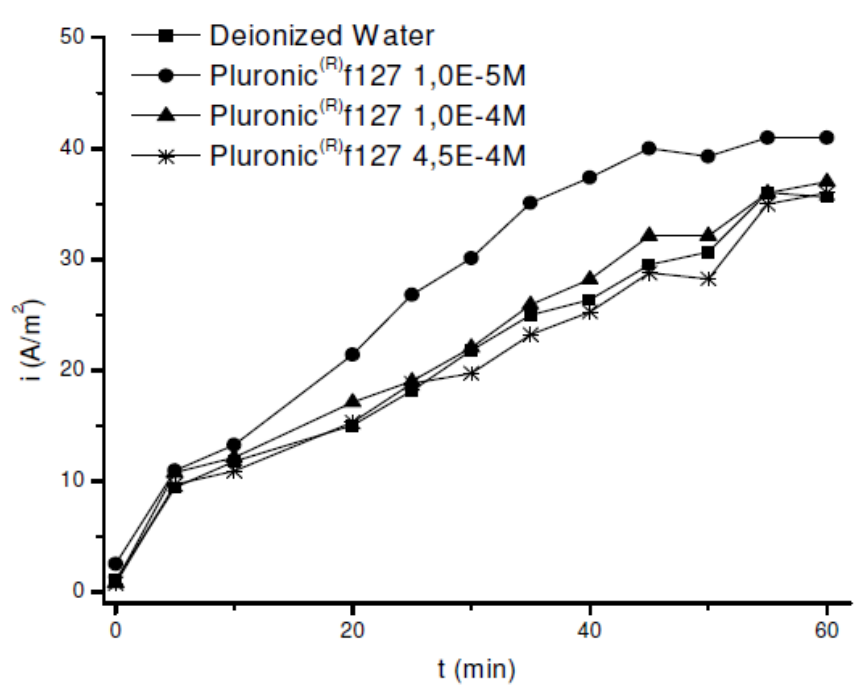

Figure 1. Current-time profile obtained for the synthesis in deionized water and at different surfactant concentrations.

On the other hand, the current time profile for all the systems presents similar shape, therefore, it can be considered that the nonionic surfactant does not modify substantially the medium conductivity. In addition, the tendency between 
Pluronic $®$ and deionized water systems could indicate that the surfactant does not react on the electrode because the current intensity, which depends on the amount of transferred electrons in the reaction, is similar in the three different concentrations of surfactant.

\section{AgNP characterization}

After the synthesis, the PSD was determined. These results are shown in Fig. 2. The figure shows a monomodal distribution for all evaluated systems except for that one with a surfactant concentration of $4.5 \times 10^{-4} \mathrm{M}$, even though, this distribution is approximately monomodal.

The best PSD was obtained when a concentration of $1.0 \times 10^{-5} \mathrm{M}$ of surfactant was used. This distribution varies between 20 to $200 \mathrm{~nm}$ with a media of $70 \mathrm{~nm}$. The results for higher concentrations showed particles that should be in nanometric range but with particle size media larger than $100 \mathrm{~nm}$. For these systems, ultrasound bath was used during 10 minutes and after that, PSD was measured again, obtaining a better size distribution and media size for nanoparticles. This behavior could be attributed to depletion flocculation phenomenon, which is typical for systems in which stabilized particles ratio is lower than the surfactant. The excess of surfactant conforms micelles, which exert localized pressure onto stabilized particles promoting flocculation processes.

For non-stabilized system, measured PSD showed particles with sizes above $1000 \mathrm{~nm}$ and the final suspension presented great instability.

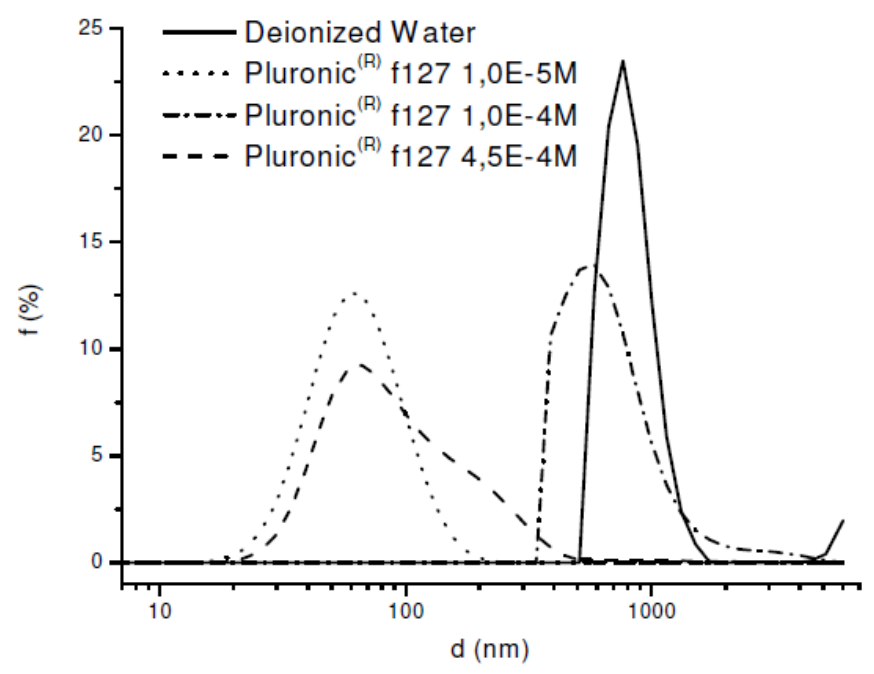

Figure 2. Particle size distribution for AgNP synthesized in different electrolytes.

Followed PSD determination, SEM microscopy was run for stabilized systems to assess morphologies and to check the agglomerates formation when surfactant was used. For the systems with Pluronic $\AA$ concentration of $1.0 \times 10^{-5}$ and $4.5 \times 10^{-4}$ $\mathrm{M}$, it was not possible to obtain images with a good definition because the resolution of SEM did not allow viewing particles less than $300 \mathrm{~nm}$.

Depletion flocculation phenomenon can be seen in micrographs obtained for the system with a surfactant concentration of $1.0 \times 10^{-4} \mathrm{M}$ (Fig. 3). These images show the agglomerate formation of different sizes and the presence of surfactant 
around the particles forming a gel type structure. The particle sizes were in accord with the PSD obtained for this system (between 300 and $1000 \mathrm{~nm}$ ).
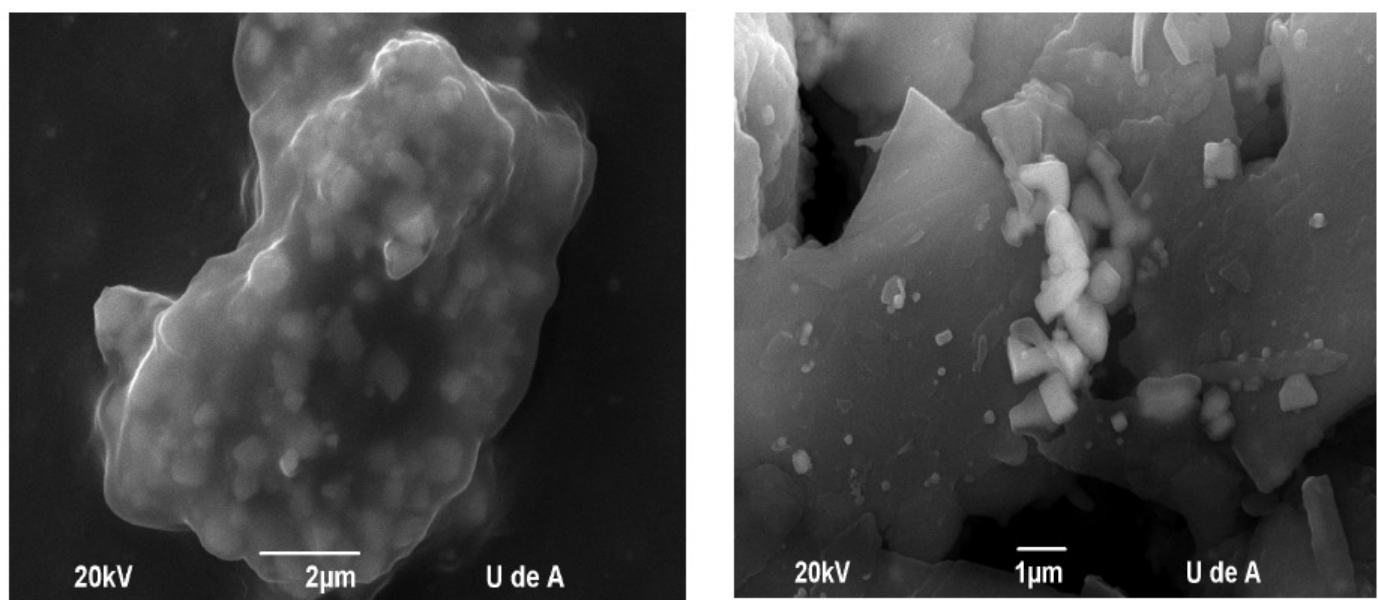

Figure 3. Particle micrographs obtained for AgNP synthesized in presence of surfactant at a concentration of $1.0 \times 10^{-4} \mathrm{M}$.

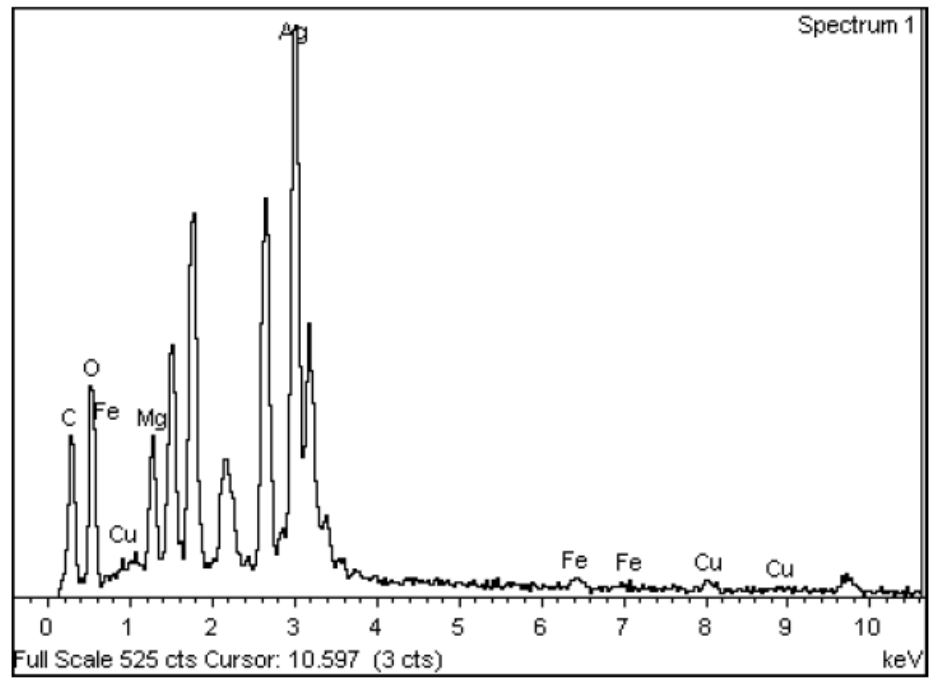

Figure 4. EDX for silver nanoparticles obtained in presence of Pluronic ${ }^{\circ}$ f127 surfactant. The non-assigned peaks are from unknown origin.

EDX analysis was run to verify the composition of synthesized nanoparticles. The obtained diffractogram is shown in Fig. 4. It can be seen that particles obtained are mainly composed of silver. The carbon and oxygen signals are due to the surfactant, while copper, iron, and magnesium should come from minor percents on silver wire.

\section{Reaction synthesis monitoring}

Once feasibility to use this electrochemical method for AgNP synthesis was found, the system selected was $1.0 \times 10^{-5} \mathrm{M}$ surfactant concentration in order to follow the reaction by UV spectroscopy. The results are shown in Figure 5. Theoretical and experimental works show that although the absorption bands are not enough as conclusive about PSD and colloidal system polidispersity, it exists 
a correlation between bandwidth and the maximum absorbance with factors like morphology and size particle polidispersity for AgNP [11,19,20]. The spectrum shows some typical bands that appear below $300 \mathrm{~nm}$ and are attributed to $\mathrm{Ag}_{4}{ }^{+}$ clusters formation. Moreover, the AgNP characteristic absorption band begins to appear at 36 minutes with a maximum between 540 and 560, which is a different value from the literature reported and coincident with the time in which the suspension starts to become gray.

However, when the synthesis progresses, it can be seen that the maximum absorbance takes values to shorter wavelengths. For the sample measured at 48 minutes, the maximum absorbance was between 470-490 $\mathrm{nm}$ and for the sample measured at 60 minutes, it was between $450-460 \mathrm{~nm}$. The left picture in Figure 5 shows the progressive change of the color of the suspension over time. The first three samples are apparently colorless, but from the fourth sample, it is possible to see a darkness of suspension, which supports the remarks from UV-Vis studies.

The spectroscopic monitoring of the reaction synthesis is related to the results obtained for the current-time profiles (Fig. 1), in which it was proposed that in a first step, the system has a saturation of $\mathrm{Ag}^{+}$ions with a saturation time of less than 24 minutes.
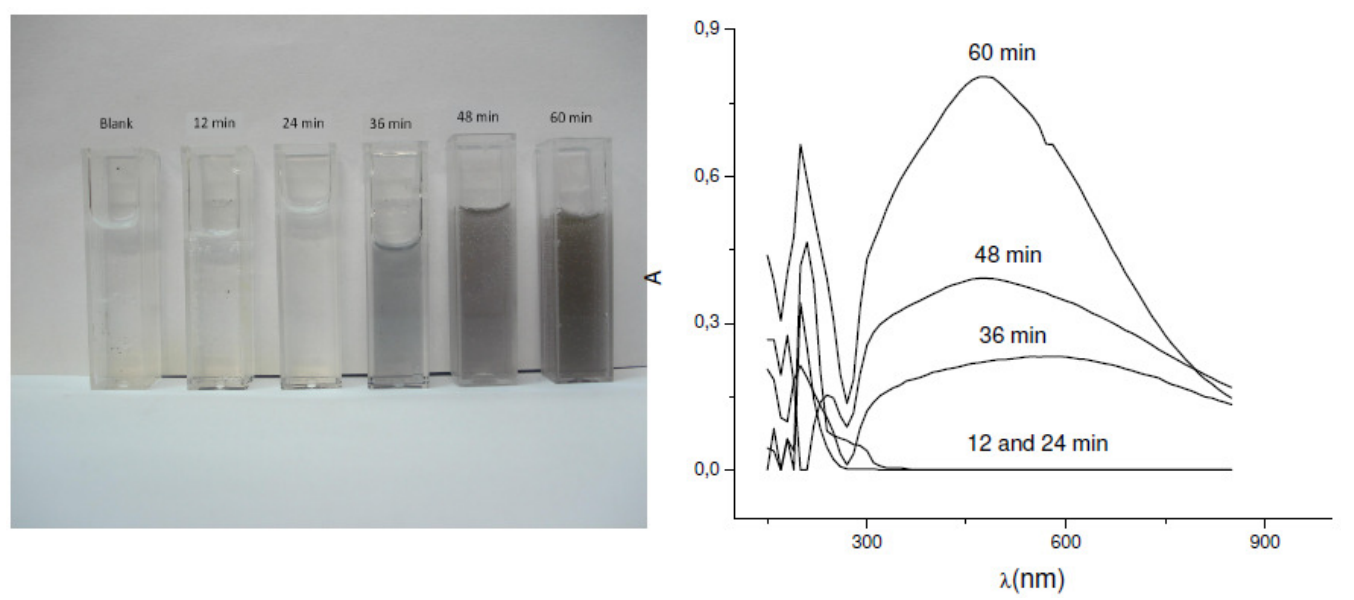

Figure 5. UV- Vis spectroscopic monitoring for AgNP's reaction synthesis.

\section{Time stability}

Fig. 6 shows variation of AgNP average diameter with time. The stability with time was analyzed for the AgNP sample synthesized at a surfactant concentration of $1.0 \times 10^{-5} \mathrm{M}$ used for PSD study. This analysis is important because the antimicrobial activity study requires ensuring time-stability for the suspended nanoparticles. The sample was stored at room temperature in absence of light for 30 days and over time and 5 measurements were accomplished. Taking into account the standard deviation obtained, it can be said that AgNP shows stability at least for the time interval in which it was monitored. 


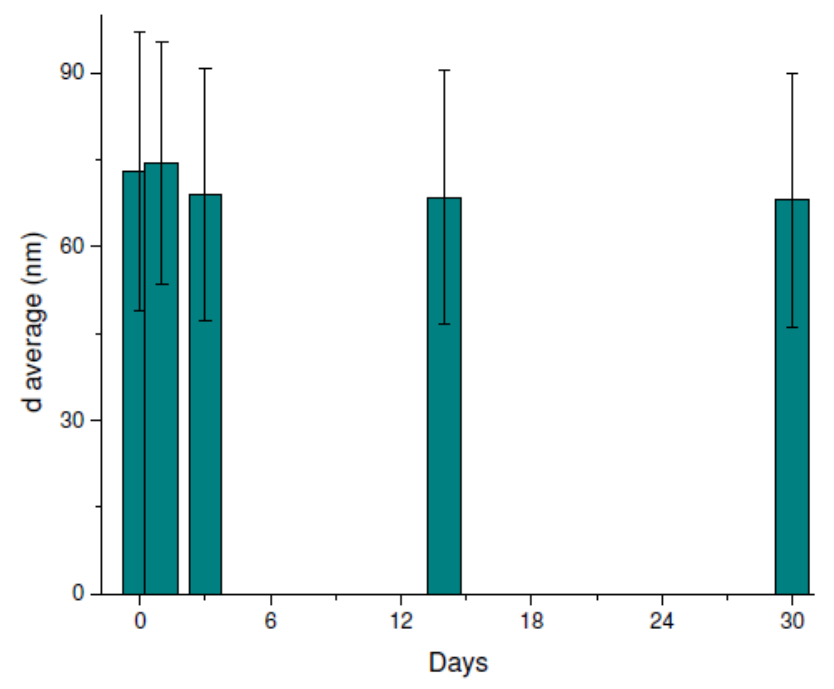

Figure 6. Variation of AgNP average diameter with time.

\section{Antimicrobial evaluation}

Table 1 shows the antimicrobial activity results for AgNP suspension electrochemically synthesized with a surfactant concentration of $1.0 \times 10^{-5} \mathrm{M}$ and without surfactant. Besides, this figure shows the activity for the same sample used in the time stability study. This results show a growth inhibition from $4 \mathrm{mg} . \mathrm{L}^{-1}$ for the suspension without surfactant, and 0.5 and $1 \mathrm{mg} . \mathrm{L}^{-1}$ for suspensions of surfactant stabilized nanoparticles on day 0 and 30 days after synthesis. These results indicate that antimicrobial activity is not affected neither by the use of surfactant nor by the aging of AgNP.

Table 1. Antimicrobial evaluation of AgNP against E. coli.

\begin{tabular}{cc}
\hline Antimicrobial Agent & MIC (mg.L) \\
\hline Culture medium and microorganism & $>16$ \\
AgNP without surfactant & 2 \\
AgNP with surfactant & 1 \\
Time effect in nanoparticles & 2 \\
Positive control (chloramphenicol) & $<1$ \\
\hline
\end{tabular}

\section{Conclusions}

It should be noted that although the electrochemical method employed for the synthesis of nanoparticles is simple and requires little complex instrumentation, it allows obtaining silver nanoparticles with a good distribution and average size. However, to ensure stability and good PSD, it is necessary to use surfactants. PSD results indicate that the use of surfactant Pluronic $₫$ f127 shows being quite efficient in the stabilization of AgNP and given its condition of biocompatible surfactant the Pluronic $\AA$ is optimal for antimicrobial activity studies because it is possible to ensure that the growth inhibition is due to the action of silver nanoparticles only. 
The AgNP shows a good antimicrobial behavior with a satisfactory minimal inhibitory concentration and the presence of surfactant seems not to affect the inhibitory activity of the nanoparticles.

The experimental results obtained are useful to design a system where immobilized AgNP could be applied for use as an antimicrobial agent.

\section{Electrochemical Synthesis of Silver Nanoparticles and its Potential use as Antimicrobial Agent: a case study on $E$. coli}

\section{Resumo}

Nanopartículas de prata (AgNP) foram sintetizadas pelo método eletroquímico usando prata metálica de alta pureza na presença do surfactante Pluronic ${ }^{\circledR}$ F127. As nanopartículas sintetizadas foram caracterizadas através de espectroscopia UV-Vis, dispersão de luz dinâmico (DLS), espectroscopia de dispersão de Raio$\mathrm{X}$ (XDS) e Microscopia Eletrônica de Varredura (MEV). A atividade antimicrobiana de AgNP foi avaliada contra Escherichia Coli.

$\mathrm{Na}$ presença de surfactante, as nanopartículas preparadas levaram a uma monomodal distribuição com um tamanho médio inferior a $100 \mathrm{~nm}$. A concentração inibitória mínima (MIC) da suspensão resultante foi avaliada através do método de microdiluição e os resultados foram satisfatórios.

\section{Acknowledgments}

We would like to thank The Committee for Research Development -CODI- and sustainability program 2011-2012 from University of Antioquia for its financial support for this research. Additionally we are grateful to Cyted program for their support for the mobility of researchers.

\section{References}

1. $\quad$ R. Bhattacharya, P. Mukherjee, Adv. Drug Delivery Rev. 60 (2008) 12891306.

2. Y.J. Nam, J.R. Lead, Sci. Total Environ. 400 (2008) 396 - 414.

3. T. Pradeep, Anshup, Thin Solid Films 517 (2009) 6441-6478.

4. I. Sondi, B. Salopek-Sondi, J. Colloid Interface Sci. 275 (2004) 177-182.

5. L.F. Espinosa-Cristóbal, G.A. Martínez-Castañón, R.E. Martínez-Martínez, J.P. Loyola-Rodríguez, N. Patiño-Marín, J.F. Reyes-Macías, F. Ruiz, Mater. Lett. 63 (2009) 2603-2606.

6. A. Nanda, M. Saravanan, Nanomed. Nanotechnol. Biol. Med. 5 (2009) 452456.

7. M. Rai, A. Yadav, A. Gade, Biotechnol. Adv. 27 (2009) 76-83.

8. V.K. Sharma, R.A. Yngard, Y. Lin, Adv. Colloid Interface Sci. 145 (2009) 83-96.

9. $\quad$ K.H. Cho, J.E. Park, T. Ozaka, S.G. Park, Electrochim. Acta 51 (2005) 956960. 
10. G. Abate, R.N. Mshana, H. Miorner, Int. J. Tuberc. Lung D 12 (1998) 1011-1016.

11. S.K. Mehta, S. Chaudhary, M. Gradzielski, J. Colloid Interface Sci. 343 (2010) 447-453.

12. W. Zhang, X. Qiao, J. Chen, Mater. Sci. Eng. B 142 (2007) 1-15.

13. M.T. Reetz, W. Helbig, J. Am. Chem. Soc. 116 (1994) 1401-1402.

14. M. Starowicz, B. Stypuła, J. Banas, Electrochem. Commun. 8 (2006 ) 227230.

15. B. Yin, H. Ma, S. Wang, S. Chen, J. Phys. Chem. B 107 (2003) 88988904.

16. R.A. Khaydarov, R.R. Khaydarov, O. Gapurova, Y. Estrin, T. Scheper, J. Nanopart. Res. 11 (2009) 1193-1200.

17. T.I. Abdullin, O.V. Bondar, Y.G. Shtyrlin, M. Kahraman, M. Culha, Langmuir 26 (2010) 5153-5159.

18. N. Kiratzis, M. Faers, P.F. Luckham, Colloids Surf. A 151 (1999) 461-471.

19. D.K. Bhui, H. Bar, P. Sarkar, G.P. Sahoo, S.P. De, A. Misra, J. Mol. Liq. 145 (2009) 33-37.

20. W.Z. Zhang, X.L. Qiao, J.G. Chen, Mater. Chem. Phys. 109 (2008) 411416. 